\title{
Dermatitis due to Unspecified Substance Taken Internally
}

National Cancer Institute

\section{Source}

National Cancer Institute. Dermatitis due to Unspecified Substance Taken Internally. NCI Thesaurus. Code C35224.

Dermatitis caused by an allergic reaction to an unspecified ingested substance. 